\title{
In-situ electropolymerized bipolar organic cathode for stable and high-rate lithium-ion batteries
}

\author{
Wei Wang, Chen Zhao, Jixing Yang, Peixun Xiong, Hai Su and Yunhua Xu*
}

\begin{abstract}
To address the dissolution issue and enhance the electrochemical performance of organic electrode materials, herein, a bipolar organic cathode was prepared by in-situ electropolymerization of amino-phenyl carbazole naphthalene diimide (APCNDI). APCNDI is composed of n-type 1,4,5,8naphthalene tetracarboxylic diimide that stores $\mathrm{Li}$ cations and p-type carbazole groups which react with anions and serve as polymerization sites. Electropolymerization completely eliminated the dissolution problem of APCNDI, and the electropolymerized cathode demonstrated a bipolar reaction with excellent electrochemical performance, stable cycling performance with a capacity retention of $92 \mathrm{~mA} \mathrm{~h} \mathrm{~g}^{-1}$ after 1000 cycles, and a superior rate performance of $72 \mathrm{~mA} \mathrm{~h} \mathrm{~g}^{-1}$ at $10 \mathrm{~A} \mathrm{~g}^{-1}$. The bipolar feature and reactions of APCNDI were systematically investigated and verified by multiple characterization techniques. Our findings provide a novel strategy for the design and fabrication of electrodes for high-performance organic batteries.
\end{abstract}

Keywords: lithium-ion battery, bipolar organic cathode, in-situ electropolymerization, polyimide, carbazole

\section{INTRODUCTION}

Redox-active organics have attracted significant research interest as promising electrode materials for lithium-ion batteries (LIBs) owing to their several merits including abundant resources of raw materials and structural versatility, which afford considerable potential for the development of sustainable batteries [1-3]. Unlike inorganic electrode materials that are prepared using limited mineral resources, redox-active organic materials are mainly fabricated employing unlimited resources such as $\mathrm{C}, \mathrm{H}$, $\mathrm{N}$, and $\mathrm{O}$ [2,4-7]. Moreover, some organic compounds can be directly obtained from natural biomass via environmentally benign production methods [8]. From the viewpoint of molecular structure design, organic com- pounds have diverse structures and can be functionalized with various groups. Therefore, organic compounds are promising candidates for electrode materials with high electrochemical performance including high specific capacity and high rate capability [2]. Additionally, organic materials have flexible structures and generally contain multiple active sites in one unit, which enable versatile storage reactions, particularly for beyond LIBs chemistries [2,9]. Clearly, organic materials offer a platform to realize novel battery technologies that may replace LIBs.

Organic electrode materials are mainly categorized into three types based on the redox reaction mechanism: ntype, p-type, and bipolar [10-13]. The n-type materials react with $\mathrm{Li}^{+}$cations, and the positive charges are compensated by the uptake of the same number of electrons, leading to a reduction reaction. This n-type reaction generally results in high specific capacity at a low voltage $(<3 \mathrm{~V})$, which is not favorable for high-energy-density batteries [2]. Representative n-type materials are carbonyl organics $[14,15]$, which are the most investigated organic electrode materials. For example, $p$-benzoquinone has a theoretical specific capacity of $496 \mathrm{~mA} \mathrm{~h} \mathrm{~g}^{-1}$ [16], and Lu et al. [17] reported a cyclohexanehexone cathode material that exhibits a high capacity of $902 \mathrm{~mA} \mathrm{~h} \mathrm{~g}^{-1}$. However, these organic molecules are highly soluble in organic liquid electrolytes, causing a fast capacity decay [16,17]. In contrast, p-type materials can accept anions and transform from neutral to positively charged states [18,19]. A p-type reaction typically occurs at a high voltage of $>3 \mathrm{~V}$ and has fast reaction kinetics owing to the lack of desolvation that commonly takes place on the anode side. However, it often delivers a low capacity, which needs to be addressed for future applications of p-type materials. Contrarily, bipolar molecules contain both $\mathrm{n}$ - and p-type parts and can react with both cations and anions [20-23], which may exhibit the combined merits (namely, high

School of Materials Science and Engineering, Key Laboratory of Advanced Ceramics and Machining Technology (Ministry of Education) and Tianjin Key Laboratory of Composite and Functional Materials, Tianjin University, Tianjin 300072, China

* Corresponding author (email: yunhua.xu@tju.edu.cn) 
capacity, high voltage, and fast kinetics) of both n- and ptype parts. They can be used as both cathode and anode depending on the potential level. Based on this concept, all-organic symmetric batteries have been developed using bipolar compounds [21]. However, the specific capacity of these batteries is low because only a single type of reaction occurs on each side, leading to limited energy density [21]. Recently, bipolar compounds were employed as cathode materials to achieve high energy density. Enhanced specific capacities were obtained with insufficient cycling stability [22,23].

Dissolution and insulating problems are intrinsic drawbacks of redox-active organic compounds $[1,2,24]$. Although many efforts have been made to overcome these issues, these problems still persist. Among the reported strategies, the use of polymers [25-29] is the most effective approach to address the dissolution issue; nevertheless, polymers suffer from severe aggregation during electrode preparation, thereby resulting in poor kinetics, incomplete reaction, and inferior electrochemical performance $[19,27,28]$. Completely utilizing the advantages of organic electrode materials is still a significant challenge. Recently, a novel preparation method, namely, insitu electropolymerization has been reported for the fabrication of polymer electrodes [30,31]. Traditional methods of polymer electrode fabrication require presynthesized polymer materials, and it is difficult to realize uniform distribution of these materials and conductive agents. However, electropolymerization involves the use of small molecules, which can improve the dispersion of organic molecules in conductive agents [32-36]. Polymerization of organic molecules and charging of the battery occurred simultaneously, the dissolution problem was completely solved, and the battery exhibited exceptional performance with stable cycling ability and fast rate capability. This shows that in-situ electropolymerization is a quite promising method for the construction of highperformance Li-organic batteries.

Herein, a bipolar polymer cathode was fabricated by insitu electropolymerization. Amino-phenyl carbazole naphthalene diimide (APCNDI) comprising p-type carbazole units and n-type 1,4,5,8-naphthalene tetracarboxylic diimide (NTCDI) units was employed as a small molecule precursor. The n-type units offer a high theoretical capacity of approximately $200 \mathrm{~mA} \mathrm{~h} \mathrm{~g}^{-1}$, and the p-type units function as not only high-voltage reaction sites, but also polymerization agents to produce a polymer structure. In-situ electropolymerization of polymer cathode completely solved the dissolution problem, and the electropolymerized cathode demonstrates ex- ceptional cycling stability and suitable reaction kinetics, thus delivering a high reversible capacity of $141 \mathrm{~mA} \mathrm{~h} \mathrm{~g}^{-1}$ at a current density of $0.1 \mathrm{~A} \mathrm{~g}^{-1}$, an excellent rate performance of $72 \mathrm{~mA} \mathrm{~h} \mathrm{~g}^{-1}$ at a high rate of $10 \mathrm{~A} \mathrm{~g}^{-1}$, and stable cycling performance over 1000 cycles with a capacity retention of $92 \mathrm{~mA} \mathrm{~h} \mathrm{~g}^{-1}$. Results reveal that the $\mathrm{p}$ type reaction enables the electropolymerization of the organic cathode, while severe dissolution problem persists when only the n-type reaction occurs.

\section{EXPERIMENTAL SECTION}

\section{Materials}

Carbazole (99\%), Pd (containing $10 \mathrm{wt} \%$ charcoal, denoted as $\mathrm{Pd} / \mathrm{C}, 99 \%)$, 4-fluoronitrobenzene (98\%), and anhydrous potassium carbonate $\left(\mathrm{K}_{2} \mathrm{CO}_{3}, 99 \%\right)$ were purchased from Innochem Co., Ltd. 1,4,5,8-Naphthalene tetracarboxylic acid dianhydride (NTCDA, 99\%), hydrazine monohydrate $\left(\mathrm{N}_{2} \mathrm{H}_{4} \cdot \mathrm{H}_{2} \mathrm{O}, 98 \%\right)$, anhydrous ethanol (EtOH, 99.7\%), anhydrous dimethyl sulfoxide (DMSO, 99.5\%), and anhydrous $N, N$-dimethylformamide (DMF, 99.5\%) were obtained from Energy Co., Ltd. Anhydrous methanol $(\mathrm{MeOH}, \mathrm{AR})$ was procured from Tianjin Komiou Chemical Reagent Co., Ltd. Graphene was purchased from Changzhou Sixth Element Technology Co., Ltd.

\section{Synthesis}

\section{Synthesis of 9-(4-nitrophenyl)-9H-carbazole}

Carbazole $(1.67 \mathrm{~g}, 10 \mathrm{mmol})$, anhydrous $\mathrm{K}_{2} \mathrm{CO}_{3}(2.76 \mathrm{~g}$, $20 \mathrm{mmol})$, and 4-fluoronitrobenzene $(1.55 \mathrm{~g}, 11 \mathrm{mmol})$ were added to a $50-\mathrm{mL}$ round-bottom flask. Then, $15 \mathrm{~mL}$ of anhydrous DMSO was added to the abovementioned solution followed by stirring for $20 \mathrm{~min}$. Subsequently, the temperature was raised to $160^{\circ} \mathrm{C}$. The reaction was performed under an inert atmosphere for $10 \mathrm{~h}$, and then, the reaction mixture was cooled to room temperature and poured into $200 \mathrm{~mL}$ of ice water followed by stirring for $20 \mathrm{~min}$. The crude product was washed with anhydrous $\mathrm{MeOH}$ and then filtered through membranes. The reaction yield was $96 \%$. The obtained product was a yellowish crude powder. ${ }^{1} \mathrm{H}$ nuclear magnetic resonance (NMR) $\left(400 \mathrm{MHz}, \mathrm{CDCl}_{3}, 298 \mathrm{~K}\right) \delta: 8.50(\mathrm{t}, 1 \mathrm{H}), 8.48(\mathrm{t}, 1 \mathrm{H})$, $8.16(\mathrm{t}, 1 \mathrm{H}), 8.14(\mathrm{t}, 1 \mathrm{H}), 7.82(\mathrm{t}, 1 \mathrm{H}), 7.81(\mathrm{t}, 1 \mathrm{H})$, 7.51-7.43 (m, $4 \mathrm{H}), 7.37-7.33(\mathrm{~m}, 2 \mathrm{H})$.

\section{Synthesis of 4-(9H-carbazol-9-yl)aniline}

Under an inert atmosphere, 9-(4-nitrophenyl)-9H-carbazole $(0.72 \mathrm{~g}, 2.5 \mathrm{mmol})$ and $10 \% \mathrm{Pd} / \mathrm{C}(21 \mathrm{mg})$ were 
added to a $50-\mathrm{mL}$ round-bottom flask followed by the introduction of absolute ethanol $(15 \mathrm{~mL})$ and thorough stirring. Then, the reaction temperature was raised to $90^{\circ} \mathrm{C}$. After the solvent was refluxed, $0.6 \mathrm{~mL}$ of anhydrous hydrazine hydrate was added to the reaction mixture to reduce the nitro group to the amino group. The reaction mixture was refluxed for $9 \mathrm{~h}$ and the hot reaction solution was filtered to remove $\mathrm{Pd} / \mathrm{C}$. Finally, the filtrate was subjected to rotary evaporation to remove the solvent. Consequently, a brown viscous liquid was acquired. The reaction yield was $94 \% .{ }^{1} \mathrm{H}$ NMR $\left(400 \mathrm{MHz}, \mathrm{d}^{6}\right.$-DMSO, $298 \mathrm{~K}) \delta: 8.21(\mathrm{~d}, 2 \mathrm{H}), 7.40(\mathrm{t}, 2 \mathrm{H}), 7.27-7.22(\mathrm{~m}, 4 \mathrm{H})$, 7.19 (d, 2 H), 6.81 (d, 2 H), 5.43 (s, 2 H).

\section{Synthesis of APCNDI}

The abovementioned brown viscous liquid $(0.61 \mathrm{~g}$, $2.35 \mathrm{mmol})$ and excess NTCDA $(0.29 \mathrm{~g}, 1.07 \mathrm{mmol})$ were added to a $50-\mathrm{mL}$ round-bottom flask, followed by the introduction of $15 \mathrm{~mL}$ of DMF. The reaction temperature was raised to $160^{\circ} \mathrm{C}$, and the mixture was refluxed for $16 \mathrm{~h}$ under an inert atmosphere. After the reaction mixture was cooled to room temperature, the acquired precipitate was washed with DMF, and suction filtration was conducted to obtain red powders. The reaction yield was $70 \%$.

\section{Characterization}

Solid-state ${ }^{13} \mathrm{C}$ NMR spectra were recorded using a Bruker NMR spectrometer. Chemical shifts were reported in ppm relative to the internal standard tetramethylsilane for ${ }^{13} \mathrm{C}$ NMR. ${ }^{1} \mathrm{H}$ NMR spectra were recorded on a Bruker $400 \mathrm{MHz}$ spectrometer at room temperature. Chemical shifts for protons in all the spectra were referred to as the residual proton resonance of $\mathrm{d}^{6}$-DMSO $(\delta: 2.54 \mathrm{ppm})$ and deuterated chloroform $\left(\mathrm{CDCl}_{3}, \delta: 7.26 \mathrm{ppm}\right)$. Morphologies and structures of the cathodes were investigated by scanning electron microscopy (SEM, S-4800 instrument). $\mathrm{X}$-ray diffraction $(\mathrm{XRD})$ patterns were acquired via Rigaku Ultima IV using $\mathrm{Cu} \mathrm{Ka}$ radiation $(\lambda=1.5406 \AA$, $40 \mathrm{kV}$, and $40 \mathrm{~mA}$ ). Fourier transform infrared (FTIR) spectra were recorded in the attenuated total reflectance mode in the range of $4000-400 \mathrm{~cm}^{-1}$ using a Bruker Alpha II FTIR spectrometer. Ultraviolet-visible spectroscopy (UV-vis, Shimadzu UV-3600 plus, Japan) and Xray photoelectron spectroscopy (XPS, K-Alpha, UK) were conducted to analyze the chemical compositions of the pristine and cycled cathodes. The cycled cathodes used for characterization were washed with diethyl carbonate (DEC) and dried. Thermogravimetric analysis (TGA) was performed using a thermogravimetric analyzer (STA
449F3 Instruments, Germany) at a heating rate of $10^{\circ} \mathrm{C} \mathrm{min}^{-1}$ under $\mathrm{N}_{2}$ atmosphere. Gaussian $09 \mathrm{~W}$ program was used to conduct density functional theory (DFT) calculations. Geometric optimization was performed based on the theoretical level of B3LYP by standard gradient techniques, which employ $6-31+\mathrm{G}(\mathrm{d})$ as the basic set.

\section{Electrochemical measurements}

APCNDI, graphene, and polyvinylidene fluoride binder were mixed at a weight ratio of $6: 3: 1$, followed by the addition of a certain amount of $N$-methyl pyrrolidone solvent to form a slurry; subsequently, this slurry was coated on a carbon-coated aluminum foil. The resulting cathodes were dried in vacuum at $100^{\circ} \mathrm{C}$ for $12 \mathrm{~h}$ with a loading of $0.6-0.8 \mathrm{mg} \mathrm{cm}^{-2}$. Coin-type cells were assembled in a vacuum glove box $\left(\mathrm{O}_{2}\right.$ and $\mathrm{H}_{2} \mathrm{O}$ levels $<0.1 \mathrm{ppm}$ ) using lithium metal foil as the counter electrode, a polypropylene (PP) separator (Celgard 2500, LLC Corp., USA), and $1 \mathrm{~mol} \mathrm{~L}^{-1}$ lithium hexafluorophosphate $\left(\mathrm{LiPF}_{6}\right)$ in ethylene carbonate $(\mathrm{EC})$ and $\mathrm{DEC}$ as the electrolyte. Galvanostatic charging and discharging tests were conducted at voltage ranges of 1.5-4.4, 1.5-3.0, and 3.0-4.4 V using a Neware battery test system. Cyclic voltammetry (CV) measurements were performed employing Solartron Analytical 1400 (AMETEK, USA) at the scan rates of 0.05 and $0.1 \mathrm{mV} \mathrm{s}^{-1}$. Rate performance was examined at different current densities ranging from 0.1 to $10 \mathrm{~A} \mathrm{~g}^{-1}$. Electrochemical impedance spectroscopy (EIS) spectra were recorded using a frequency response analyzer (Solartron Analytical, 1400) coupled with an electrochemical interface (Solartron Analytical, 1470) at an amplitude of $5 \mathrm{mV}$ and in the frequency range of $0.1-100,000 \mathrm{~Hz}$.

\section{RESULTS AND DISCUSSION}

The procedure for the synthesis of APCNDI is illustrated in Schemes S1-S3 [37,38]. Herein, 9-(4-nitrophenyl)-9Hcarbazole was generated by the Ullmann condensation reaction of carbazole and 4-fluoronitrobenzene. Then, 9-(4-nitrophenyl)-9H-carbazole was reduced to $4-(9 H-$ carbazol-9-yl)aniline by hydrazine hydrate. Finally, APCNDI molecules were synthesized in an acceptable yield via a dehydration condensation reaction of $4-(9 H-$ carbazol-9-yl)aniline and NTCDA. A single APCNDI molecule comprises four n-type carbonyl groups, which can reversibly store alkali metal cations and a couple of p-type carbazole groups that can adsorb anions (Fig. 1a). The chemical structure of APCNDI was confirmed by FTIR and solid-state ${ }^{13} \mathrm{C}$ NMR spectroscopies (Fig. S1). 
a

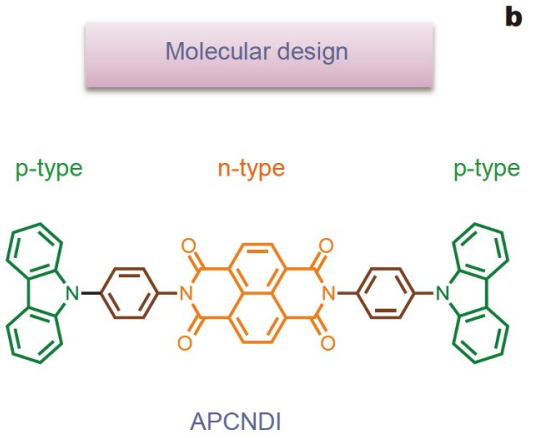

b

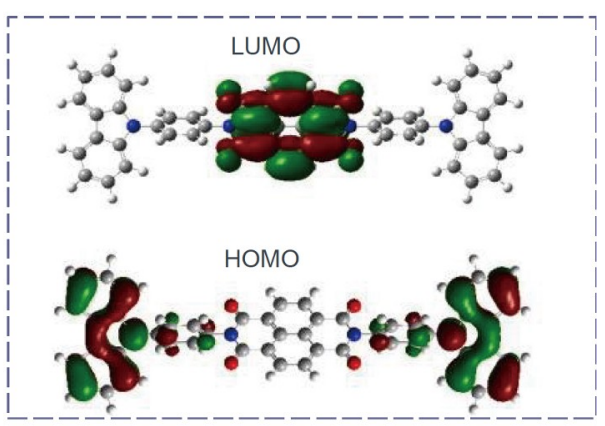

c

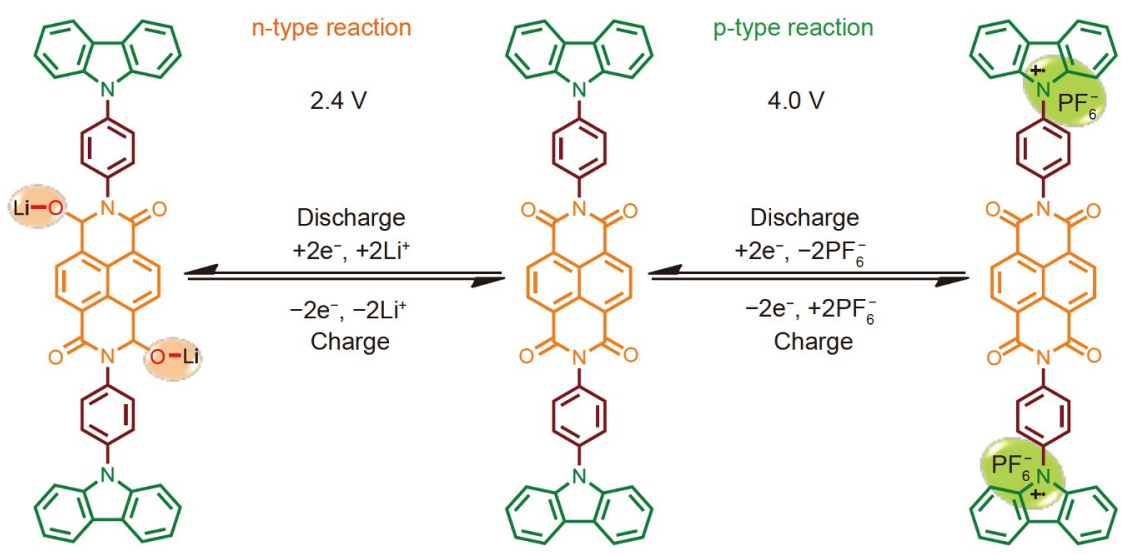

Figure 1 (a) Molecular structure, (b) HOMO and LUMO energy levels, and (c) n-type and p-type reactions of APCNDI.

The characteristic peaks located at 1714 and $1670 \mathrm{~cm}^{-1}$ are ascribed to asymmetric and symmetric stretching vibration peaks of the carbonyl groups in NTCDI, respectively. The peak of the $-\mathrm{C}-\mathrm{N}$ bond in NTCDI appears at $1335 \mathrm{~cm}^{-1}$, and the absorption peak at $1242 \mathrm{~cm}^{-1}$ is attributed to the stretching vibration of the $-\mathrm{C}-\mathrm{N}$ bond in the carbazole groups [38]. These characteristic peaks confirm the co-existence of carbonyl groups and two types of $\mathrm{C}-\mathrm{N}$ bonds in APCNDI. The ${ }^{13} \mathrm{C}$ NMR spectrum shows characteristic chemical shifts at 162 and $141 \mathrm{ppm}$ corresponding to the $\mathrm{C}$ atoms in the carbonyl groups of NTCDI and in the $\mathrm{C}-\mathrm{N}$ bonds of the carbazole groups [39]. The absorption peaks in the UV-vis spectra are consistent with the results reported in the Ref. [38] (Fig. S2). The abovementioned results demonstrate the successful synthesis of APCNDI for electropolymerization. TGA was conducted to evaluate the stability of APCNDI. Results indicate that APCNDI is very stable at ambient temperature (Fig. S3). Morphology of APCNDI was examined by SEM, showing a flaky structure with randomly distributed sizes (Fig. S4a). XRD reveals a suitable crystalline structure of APCNDI (Fig. S4b).

Distributions of the highest occupied molecular orbital
(HOMO) and lowest unoccupied molecular orbital (LUMO) of APCNDI were evaluated by DFT calculations (Fig. 1b). The LUMO of APCNDI is mainly concentrated on the NTCDI part, and the HOMO is distributed on the carbazole groups, highlighting the bipolar feature of APCNDI. The completely separate distribution of HOMO and LUMO avoids their interference with each other during the $\mathrm{n}$ - and p-type reactions, ensuring high reactivity. The $\mathrm{n}$ - and $\mathrm{p}$-type reactions have average voltages of approximately 2.4 and $4.0 \mathrm{~V} v s$. $\mathrm{Li}^{+} / \mathrm{Li}$, respectively (Fig. 1c), implying that APCNDI would exhibit two voltage plateaus and a theoretical capacity of $143 \mathrm{~mA} \mathrm{~h} \mathrm{~g}^{-1}[40,41]$.

Solubility of APCNDI in the electrolyte was examined by immersing APCNDI in $1 \mathrm{~mol} \mathrm{~L}^{-1} \mathrm{LiPF}_{6}$ in a mixed solvent of EC and DEC with a volume ratio of 1:1 for six days. The soaked electrolyte was analyzed by FTIR and ${ }^{1} \mathrm{H}$ NMR (Fig. S5). No significant difference was observed between the pristine and soaked electrolytes, and no signal of APCNDI was detected in the FTIR and ${ }^{1} \mathrm{H}$ NMR spectra. These results show that APCNDI has poor solubility in the electrolyte, which can be attributed to the large molecular conjugated system and rigid molecular 
structure of APCNDI. Organic electrode materials with poor solubility are preferred to achieve a stable cycling performance of the battery.

Electrochemical performance of APCNDI was investigated in coin-type cells. Initially, the cycling performance of APCNDI was examined in the 1.5-3.0 and 3.0-4.4 V voltage ranges, which correspond to single $\mathrm{n}$ type and p-type reactions (Fig. 2), respectively. In the low-voltage range of $1.5-3.0 \mathrm{~V}$, the galvanostatic charge/ discharge curves show typical voltage plateaus at 2.6/2.2 V, respectively, which are consistent with the CV curves (Fig. S6a) and similar to those of the NTCDI electrodes (Fig. 2a) [40]. These results are associated with the reversible transformation between enol and carbonyl structures upon the insertion/extraction of lithium ions. The initial reversible capacity of APCNDI is approximately $70 \mathrm{~mA} \mathrm{~h} \mathrm{~g}^{-1}$, close to half of the theoretical capacity (Fig. 2b), suggesting high activity of NTCDI in APCNDI. In the higher cycling voltage range of 3.0-4.4 V, a long charging plateau was obtained for the first cycle, which was attributed to the electropolymerization of APCNDI molecules under an electric field. For the second cycle, a sloping voltage plateau centered at $4.0 \mathrm{~V}$ was acquired, which resulted from the p-type reaction based on the insertion/extraction of anions into/out of the carbazole groups, respectively (Fig. 2c, d) [41]. These results are also reflected in the CV curves (Fig. S6b) and show that APCNDI has bipolar electrochemical activity as a cathode material for LIBs. It is noted that low-voltage cycling suffers a fast capacity decay, and only $37 \mathrm{~mA} \mathrm{~h} \mathrm{~g}^{-1}$ capacity was retained after 500 cycles (Fig. 2b) due to the dissolution of lithiated APCNDI molecules (Fig. 2e, f) [42]. In contrast, the capacity of APCNDI gradually increased to a stable level at 3.0-4.4 V, indicating that no dissolution occurred owing to electropolymerization (Fig. 2d). Therefore, electropolymerization plays a key role in the cycling performance of APCNDI (Fig. 2g).

Dissolution behaviors of the APCNDI cathodes, separators, and electrolytes cycled at different voltages were also explored. The cells were first cycled at $1.5-3.0$ and $1.5-4.4 \mathrm{~V}$ for 500 cycles, and then disassembled upon being charged/discharged to $3.0 \mathrm{~V}$ to retrieve the cycled samples. Fig. 3a, b show the images of the cycled separators. When the separator was cycled at $1.5-3.0 \mathrm{~V}$, it turned dark green, implying that lithiated APCNDI se-
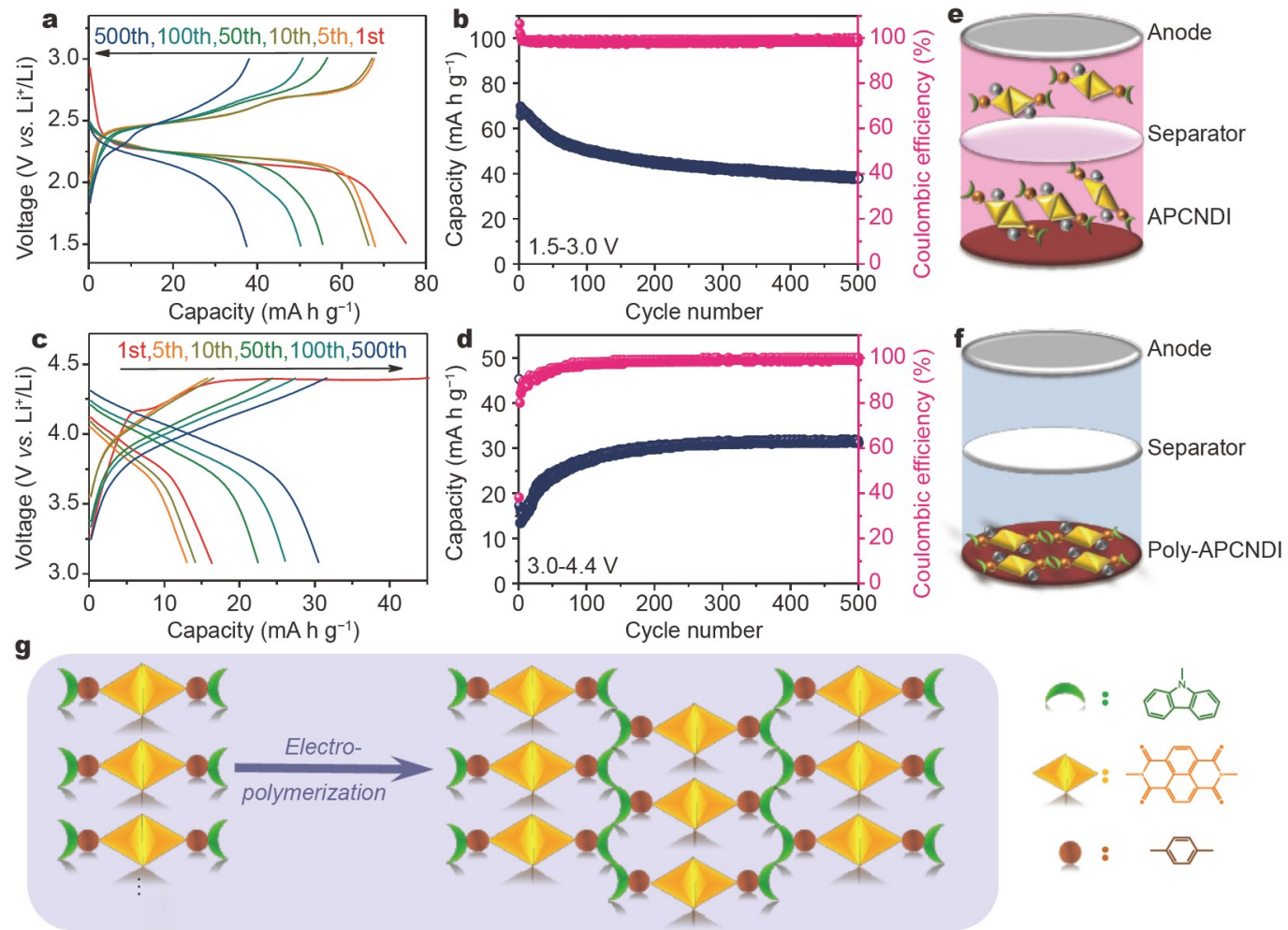

Figure 2 (a, c) Galvanostatic charge/discharge profiles of APCNDI cathode under different potential ranges at $1 \mathrm{~A} \mathrm{~g} \mathrm{~g}^{-1}$, and (b, d) cycling performance of APCNDI cathode under different potential ranges of $(a, b) 1.5-3.0 \mathrm{~V}$ and $(c, d) 3.0-4.4 \mathrm{~V}$ at $1 \mathrm{~A} \mathrm{~g}^{-1}$. Schematic illustrations of the APCNDI cathode cycling in (e) 1.5-3.0 V, (f) 3.0-4.4 V, and (g) electrochemical polymerization reaction. 

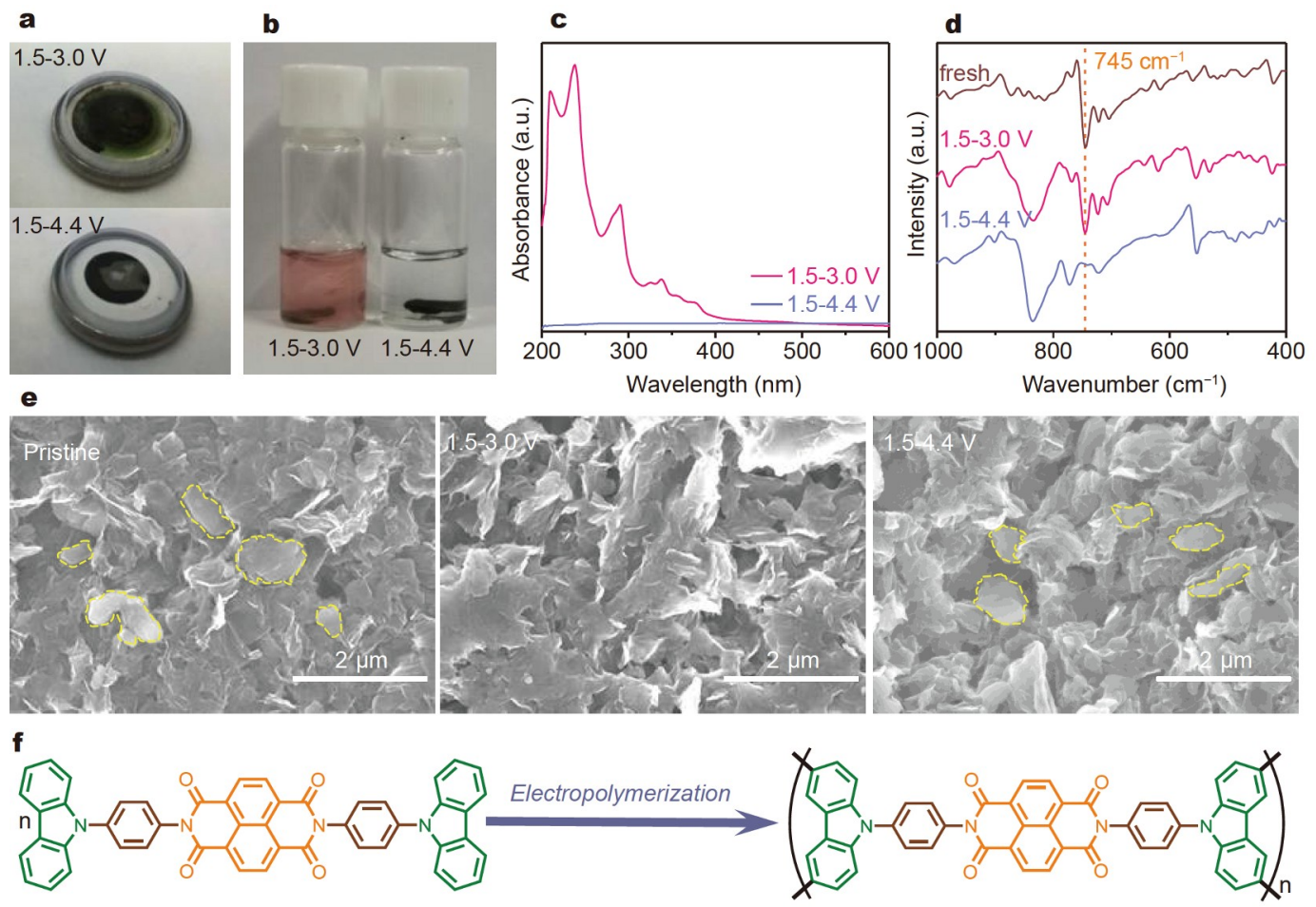

Figure 3 (a) Digital images of PP separators and (b) electrolytes soaked with APCNDI electrodes after 500 cycles at $1 \mathrm{~A} \mathrm{~g}^{-1}$ under different voltage windows. (c) UV-vis spectra, (d) FTIR spectra, and (e) SEM images of pristine and cycled APCNDI cathodes in different voltage ranges of 1.5-3.0 and 1.5-4.4 V. (f) Reaction of in-situ electropolymerization.

verely dissolved in the electrolyte. However, the separator cycled in the wider voltage range did not show any apparent change, suggesting no considerable dissolution of lithiated APCNDI. Furthermore, the cycled APCNDI cathodes were immersed in $2 \mathrm{~mL}$ of electrolyte for three days. The electrolyte soaked with the low-voltage cycled cathode turned pale pink, whereas the high-voltage cycled cathode remained colorless. Considering the red color of pristine APCNDI, the color variation of the separators shows that soluble products were generated during lowvoltage cycling, whereas no such products were produced during high-voltage cycling. To eliminate the interference from the ester group, the cathodes cycled at $1.5-3.0 \mathrm{~V}$ were soaked in $2 \mathrm{~mL}$ of pure dimethoxyethane electrolyte. FTIR spectra of the soaking solution also indicated the dissolution of the discharge products (Fig. S7). The same phenomenon was revealed by the UV-vis spectra of the soaking electrolytes (Fig. 3c). In the spectrum of the lowvoltage cycled electrolyte, characteristic absorption peaks for the $\pi-\pi^{*}$ transition of aromatic amine, $n-\pi^{*}$ transition of carbonyl groups, and the large conjugated system of the entire molecule were obtained at 238, 290, and 300-400 nm, respectively [38]. However, no absorption peak was observed in the UV-vis spectrum of the highvoltage cycled electrolyte. These results manifest that high-voltage cycling can effectively avoid the dissolution of organic electrode materials because of the electropolymerization.

FTIR spectra of the pristine and cycled cathodes at different voltage ranges prove the occurrence of electropolymerization during high-voltage cycling (Fig. 3d). A peak at $745 \mathrm{~cm}^{-1}$ was observed in the FTIR spectra of the pristine and low-voltage-cycled cathodes, which corresponds to the out-of-plane $-\mathrm{C}-\mathrm{H}$ bending vibration of bis-substituted benzene [39]. Nevertheless, it is absent for the high-voltage cycled cathodes, showing that the benzene rings in the carbazole groups varied from bis-substituted to tri-substituted, confirming the occurrence of electropolymerization at high voltages. Stability of the APCNDI cathode was also verified by comparing the morphologies of the pristine and cycled cathodes via SEM (Fig. 3e and Fig. S8). The pristine and high-voltage cycled cathodes demonstrated similar morphologies, and APCNDI particles were clearly observed, indicative of improved stability of the APCNDI cathode. However, no active material apparently presented in the case of the 
low-voltage cycled cathode because of the dissolution of the lithiated APCNDI molecules in the electrolyte. A schematic of the in-situ electropolymerization of APCNDI is shown in Fig. 3f. A cross-linked structure of APCNDI was formed during electropolymerization.

Kinetic evolution of the APCNDI cathode during cycling at different voltages was investigated by EIS. Fig. S9 shows the Nyquist plots of the APCNDI cathodes after different cycles and the fitting equivalent circuits, where $R_{\mathrm{s}}, R_{\mathrm{f}}$, and $R_{\mathrm{ct}}$ represent the bulk resistance of the electrolyte, the electrode/electrolyte interphase resistance, and the charge transfer resistance, respectively. The fitted values are summarized in Tables S1 and S2. Similar impedances were observed during the initial 10 cycles; nevertheless, substantially smaller impedances were noticed after 100 cycles in the voltage range of $1.5-4.4 \mathrm{~V}$ compared with those in the voltage range of $1.5-3.0 \mathrm{~V}$, indicating that the cathodes were effectively stabilized by electropolymerization.

Influences of the charge cut-off voltage on the electrochemical performance of APCNDI were investigated (Fig. S10). The discharge capacity gradually increased from 82 to $141 \mathrm{~mA} \mathrm{~h} \mathrm{~g}^{-1}$ as the cut-off voltage raised from 4.0 to $4.7 \mathrm{~V}$ (Fig. S10a). With the variation of the charge cut-off voltage, no significant change was observed in the low-voltage plateau corresponding to the n-type reaction, whereas the capacity contribution by the high-voltage $\mathrm{p}$ type reaction showed a gradual increase (Fig. S10b). These findings indicate that the n-type reaction is independent of the charge cut-off voltage, whereas a more complete p-type reaction occurs at a higher cut-off voltage (Fig. S10c, d). Coulombic efficiency of the APCNDI cathode significantly decreased as the cutoff voltage increased (Fig. S11a-d), which should be caused by the more severe decomposition of electrolyte at high voltages and led to poor cycling stability. SEM images of the cathodes cycled at different high cut-off voltages showed that the increase in the high cut-off voltage intensified the decomposition of the electrolyte (Fig. S11e, f).

Therefore, a mediate cut-off voltage of $4.4 \mathrm{~V}$ was used for the cycling performance test (Fig. 4). CV curves were acquired at a scan rate of $0.05 \mathrm{mV} \mathrm{s}^{-1}$ (Fig. 4a). During the first cycle, an extremely strong and sharp anodic peak near $4.4 \mathrm{~V}$ and two small sharp peaks at 2.6 and $2.1 \mathrm{~V}$ for charge and discharge were observed, respectively. The strong sharp peak was attributed to the electropolymerization of APCNDI. All the abovementioned peaks disappeared during the second cycle, implying that they were associated with electropolymerization. After the first cycle, two redox bands were obtained at approxi- mately 2.4 and $4.0 \mathrm{~V}$, corresponding to the $\mathrm{n}$ - and p-type reactions. This finding is in appropriate agreement with the bipolar structural feature of APCNDI. Electropolymerization is also indicated by the presence of a long flat charge voltage plateau above $4.2 \mathrm{~V}$ in the charge/ discharge profiles (Fig. 4b). From the second cycle onwards, no change was observed in the voltage profiles, suggesting suitable cycling stability of APCNDI. APCNDI delivered an initial reversible capacity of $118 \mathrm{~mA} \mathrm{~h} \mathrm{~g}^{-1}$, which increased to $141 \mathrm{~mA} \mathrm{~h} \mathrm{~g}^{-1}$ after 100 cycles due to the electropolymerization of APCNDI (Fig. 4c).

Rate performance of the electropolymerized cathode was examined at different current densities ranging from 0.1 to $10 \mathrm{~A} \mathrm{~g}^{-1}$ after precycling at $0.1 \mathrm{~A} \mathrm{~g}^{-1}$ (Fig. 4d). The electropolymerized cathode demonstrated an exceptional rate performance. Its initial discharge capacity was $139 \mathrm{~mA} \mathrm{~h} \mathrm{~g}^{-1}$ with a coulombic efficiency of approximately $96 \%$ at $0.1 \mathrm{~A} \mathrm{~g}^{-1}$. As the current density increased from 0.1 to $1 \mathrm{~A} \mathrm{~g}^{-1}$, the discharge capacity slightly varied from 139 to $123 \mathrm{~mA} \mathrm{~h} \mathrm{~g}^{-1}$. When the current density was up to $10 \mathrm{~A} \mathrm{~g}^{-1}$, the capacity remained at $72 \mathrm{~mA} \mathrm{~h} \mathrm{~g}^{-1}$. When the current density returned to $0.1 \mathrm{~A} \mathrm{~g}^{-1}$, the specific discharge capacity recovered to $141 \mathrm{~mA} \mathrm{~h} \mathrm{~g}^{-1}$, which demonstrated the electropolymerized cathode exhibited a better rate performance in the high-voltage range than that in the low-voltage range (Fig. S12). At all current densities, two plateaus were clearly observed, which agreed well with the bipolar feature of APCNDI (Fig. S13). At $5 \mathrm{Ag}^{-1}$, a reversible capacity of $103 \mathrm{~mA} \mathrm{~h} \mathrm{~g}^{-1}$ was achieved, and a capacity of $92 \mathrm{~mA} \mathrm{~h} \mathrm{~g}^{-1}$ was retained after 1000 cycles with a high coulombic efficiency of approximately 100\%. Moreover, the charge/ discharge curves remained almost unchanged after 1000 cycles (Fig. 4e and Fig. S14), which indicates that APCNDI has outstanding cycling stability at high current densities. Additionally, APCNDI exhibited excellent rate capability and cycle life when compared with those of other bipolar electrode materials for LIBs (Fig. S15 and Table S3).

Reaction mechanism was investigated by ex-situ FTIR and XPS spectroscopies at different charge/discharge states (Fig. 5a-c). APCNDI and the conductive agent graphene showed distinct signals from 800 to $850 \mathrm{~cm}^{-1}$ (Fig. S16), which overlapped with the characteristic peak of $\mathrm{PF}_{6}^{-}$at $830 \mathrm{~cm}^{-1}$ (Fig. S17a) [30,43]. To avoid the interference of graphene, lithium tetrafluoroborate $\left(\mathrm{LiBF}_{4}\right)$ was employed in the FTIR spectroscopy because of its characteristic infrared peak $\left(766 \mathrm{~cm}^{-1}\right)$ and similar physicochemical properties as a charger carrier in different electrolytes (Fig. S17b). Strong characteristic peak of $\mathrm{BF}_{4}{ }^{-}$ 

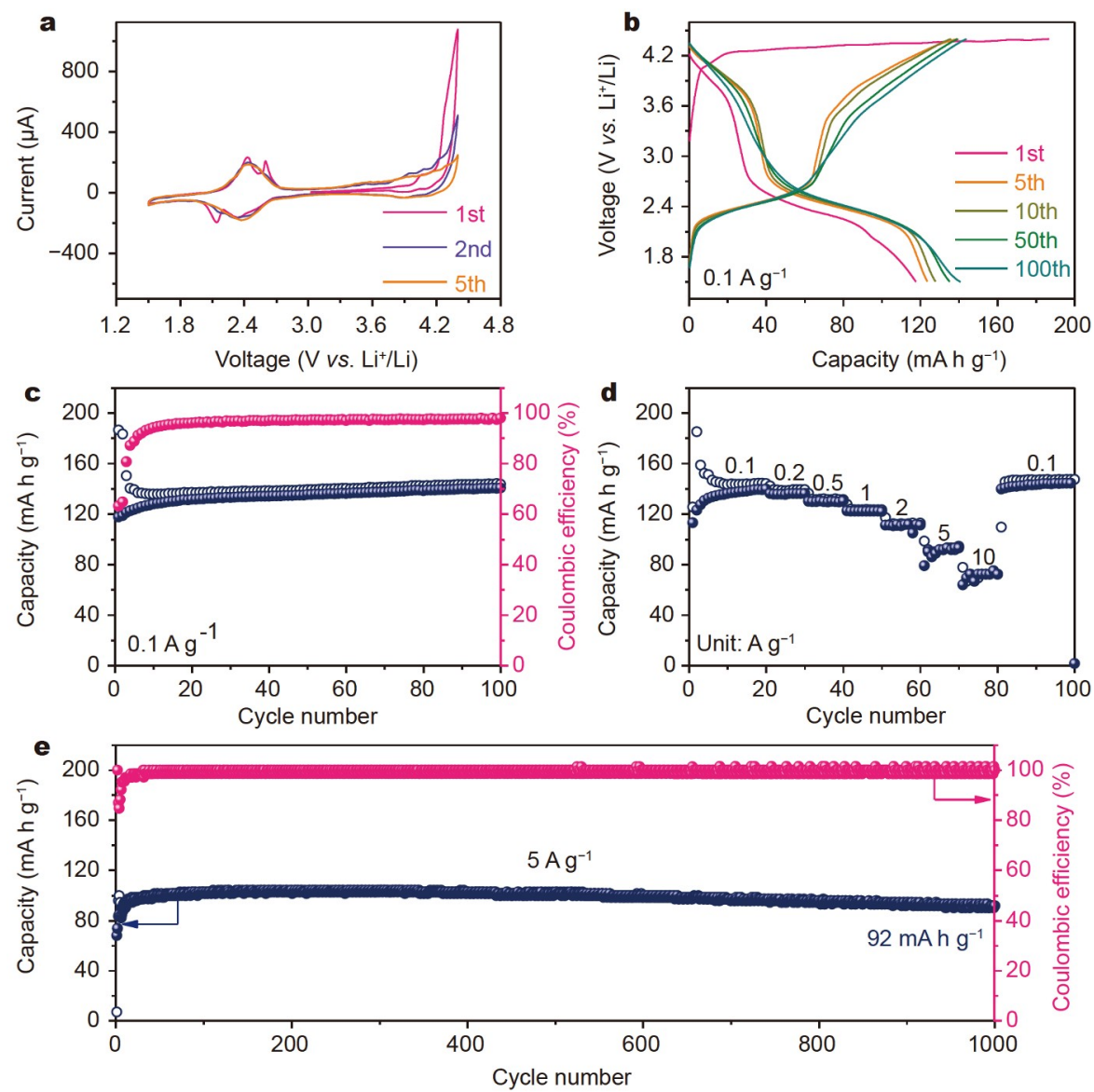

Figure 4 Electrochemical performance of APCNDI cathodes: (a) CV curves at $0.05 \mathrm{mV} \mathrm{s}^{-1}$, (b) selected charge/discharge profiles, (c) cycling performance in the voltage range from $1.5-4.4 \mathrm{~V}_{\text {at }} 0.1 \mathrm{~A} \mathrm{~g}^{-1}$, (d) rate performance, and (e) long-term cycling performance at $5 \mathrm{~A} \mathrm{~g}^{-1}$.

anions located at $766 \mathrm{~cm}^{-1}$ was clearly acquired at $4.4 \mathrm{~V}$, but it became weaker at lower voltages and disappeared below $2.5 \mathrm{~V}$, demonstrating the reversible p-type reaction of carbazole groups. Similarly, the peaks of the carbonyl groups at 770, 1714, and $1670 \mathrm{~cm}^{-1}$ and the peak of C-O$\mathrm{Li}$ at $1380 \mathrm{~cm}^{-1}$ underwent a reversible variation during charge/discharge [44], indicative of the n-type reaction of the carbonyl groups (Fig. 5b). In the full survey XPS spectrum of the pristine APCNDI cathode, four peaks were obtained at 284, 400, 532, and $687 \mathrm{eV}$, corresponding to $\mathrm{C} 1 \mathrm{~s}, \mathrm{~N} 1 \mathrm{~s}, \mathrm{O} 1 \mathrm{~s}$, and $\mathrm{F}$ 1s (Fig. S18). When the cathode was charged to $4.4 \mathrm{~V}$, a new peak appeared at $400.5 \mathrm{eV}$ in the $\mathrm{N} 1 \mathrm{~s}$ spectrum, which was attributed to $\mathrm{PF}_{6}^{-}$; it became weaker at $3.0 \mathrm{~V}$ and disappeared at $1.5 \mathrm{~V}$ [30]. The F 1s spectra at different states also confirmed the adsorption of $\mathrm{PF}_{6}{ }^{-}$at high voltages (Fig. S19). This was accompanied by the appearance of the $\mathrm{C}-\mathrm{O}-\mathrm{Li}$ peak and the increase in the intensity of the carbonyl group peaks in the O 1s XPS spectra (Fig. 5c). These results are well consistent with the FTIR results. Fig. 5d shows that a low platform originates from the reduction reaction of the n-type carbonyl groups, whereas a high platform derives from the oxidation reaction of the p-type carbazole groups, proving the bipolar electrochemical activity of APCNDI molecules. The electrochemical performance of APCNDI was investigated using different lithium salts, namely $\mathrm{LiBF}_{4}$, lithium perchlorate $\left(\mathrm{LiClO}_{4}\right)$, and lithium bis(fluorosulfonyl)imide (LiFSI). Results showed that $\mathrm{LiPF}_{6}$ delivered the best performance (Fig. S20).

\section{CONCLUSIONS}

In summary, a bipolar organic cathode was constructed by in-situ electropolymerization using APCNDI, which is composed of an n-type NTCDI part that stores $\mathrm{Li}^{+}$and $\mathrm{p}$ type carbazole groups which react with anions and serve as polymerization sites. Electropolymerization completely solved the dissolution issue of APCNDI, and the electropolymerized cathode demonstrated excellent electro- 

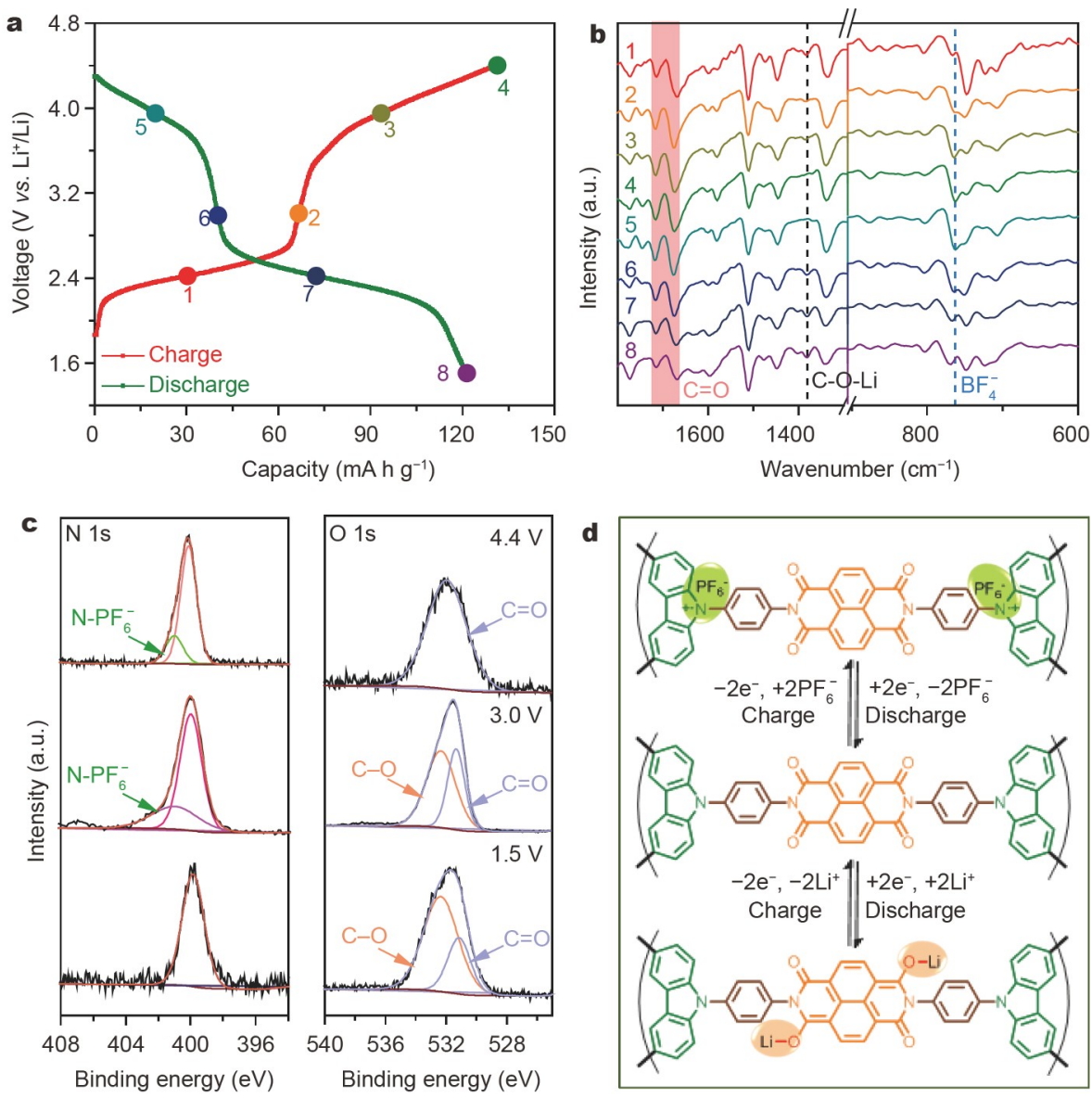

Figure 5 (a) Galvanostatic charge/discharge profiles with marked points for FTIR and XPS tests. (b) FTIR spectra and (c) high-resolution N 1s and $\mathrm{O}$ 1s spectra of APCNDI cathode at different charge/discharge states marked in (a). (d) Mechanism illustration of the n- and p-type reactions of APCNDI.

chemical performance, with cycling stability of $1000 \mathrm{cy}$ cles and a superior rate performance of $72 \mathrm{~mA} \mathrm{~h} \mathrm{~g}^{-1}$ at $10 \mathrm{~A} \mathrm{~g}^{-1}$. The bipolar feature and reactions of APCNDI were systematically investigated and verified by multiple analytical techniques. Our findings offer a new strategy for the design and fabrication of electrodes for highperformance organic batteries.

\section{Received 21 January 2021; accepted 21 April 2021; published online 25 June 2021}

1 Lu Y, Chen J. Prospects of organic electrode materials for practical lithium batteries. Nat Rev Chem, 2020, 4: 127-142

2 Lu Y, Zhang Q, Li L, et al. Design strategies toward enhancing the performance of organic electrode materials in metal-ion batteries. Chem, 2018, 4: 2786-2813

3 Fan X, Liu B, Liu J, et al. Battery technologies for grid-level largescale electrical energy storage. Trans Tianjin Univ, 2020, 26: 92103

4 Muench S, Wild A, Friebe C, et al. Polymer-based organic batteries. Chem Rev, 2016, 116: 9438-9484
5 Schon TB, McAllister BT, Li PF, et al. The rise of organic electrode materials for energy storage. Chem Soc Rev, 2016, 45: 6345-6404

6 Liang Y, Yao Y. Positioning organic electrode materials in the battery landscape. Joule, 2018, 2: 1690-1706

7 Wang $\mathrm{M}, \mathrm{Lu} \mathrm{W}$, Zhang $\mathrm{H}$, et al. Organic electrode materials for non-aqueous K-ion batteries. Trans Tianjin Univ, 2021, 27: 1-23

8 Zhang L, Liu Z, Cui G, et al. Biomass-derived materials for electrochemical energy storages. Prog Polym Sci, 2015, 43: 136-164

9 Poizot P, Gaubicher J, Renault S, et al. Opportunities and challenges for organic electrodes in electrochemical energy storage. Chem Rev, 2020, 120: 6490-6557

10 Poizot P, Dolhem F, Gaubicher J. Progress in all-organic rechargeable batteries using cationic and anionic configurations: Toward low-cost and greener storage solutions? Curr Opin Electrochem, 2018, 9: 70-80

11 Lee S, Hong J, Kang K. Redox-active organic compounds for future sustainable energy storage system. Adv Energy Mater, 2020, 10: 2001445

12 Luo C, Ji X, Hou S, et al. Azo compounds derived from electrochemical reduction of nitro compounds for high performance Liion batteries. Adv Mater, 2018, 30: 1706498

13 Luo C, Xu GL, Ji X, et al. Reversible redox chemistry of azo 
compounds for sodium-ion batteries. Angew Chem Int Ed, 2018, 57: 2879-2883

14 Häupler B, Wild A, Schubert US. Carbonyls: Powerful organic materials for secondary batteries. Adv Energy Mater, 2015, 5: 1402034

15 Zhao Q, Zhu Z, Chen J. Molecular engineering with organic carbonyl electrode materials for advanced stationary and redox flow rechargeable batteries. Adv Mater, 2017, 29: 1607007

16 Yang J, Xiong P, Shi Y, et al. Rational molecular design of benzoquinone-derived cathode materials for high-performance lithium-ion batteries. Adv Funct Mater, 2020, 30: 1909597

17 Lu Y, Hou X, Miao L, et al. Cyclohexanehexone with ultrahigh capacity as cathode materials for lithium-ion batteries. Angew Chem Int Ed, 2019, 58: 7020-7024

18 Oyaizu K, Nishide H. Radical polymers for organic electronic devices: A radical departure from conjugated polymers? Adv Mater, 2009, 21: 2339-2344

19 Janoschka T, Hager MD, Schubert US. Powering up the future: Radical polymers for battery applications. Adv Mater, 2012, 24: 6397-6409

20 Charlton GD, Barbon SM, Gilroy JB, et al. A bipolar verdazyl radical for a symmetric all-organic redox flow-type battery. J Energy Chem, 2019, 34: 52-56

21 Dai G, He Y, Niu Z, et al. A dual-ion organic symmetric battery constructed from phenazine-based artificial bipolar molecules. Angew Chem Int Ed, 2019, 58: 9902-9906

22 Liu T, Kim KC, Lee B, et al. Enhanced lithium storage of an organic cathode via the bipolar mechanism. ACS Appl Energy Mater, 2020, 3: 3728-3735

23 Wang HG, Wang H, Si Z, et al. A bipolar and self-polymerized phthalocyanine complex for fast and tunable energy storage in dual-ion batteries. Angew Chem Int Ed, 2019, 58: 10204-10208

24 Sui $\mathrm{D}, \mathrm{Xu} \mathrm{L}$, Zhang $\mathrm{H}$, et al. A 3D cross-linked graphene-based honeycomb carbon composite with excellent confinement effect of organic cathode material for lithium-ion batteries. Carbon, 2020, 157: 656-662

25 Wu J, Rui X, Wang C, et al. Nanostructured conjugated ladder polymers for stable and fast lithium storage anodes with highcapacity. Adv Energy Mater, 2015, 5: 1402189

26 Xie J, Gu P, Zhang Q. Nanostructured conjugated polymers: Toward high-performance organic electrodes for rechargeable batteries. ACS Energy Lett, 2017, 2: 1985-1996

27 Yang J, Shi Y, Sun P, et al. Optimization of molecular structure and electrode architecture of anthraquinone-containing polymer cathode for high-performance lithium-ion batteries. ACS Appl Mater Interfaces, 2019, 11: 42305-42312

28 Yang J, Shi Y, Li M, et al. Performance enhancement of polymer electrode materials for lithium-ion batteries: From a rigid homopolymer to soft copolymers. ACS Appl Mater Interfaces, 2020, 12: 32666-32672

29 Geng K, He T, Liu R, et al. Covalent organic frameworks: Design, synthesis, and functions. Chem Rev, 2020, 120: 8814-8933

30 Zhao C, Chen Z, Wang W, et al. In situ electropolymerization enables ultrafast long cycle life and high-voltage organic cathodes for lithium batteries. Angew Chem Int Ed, 2020, 59: 11992-11998

31 Zeng C, Wang B, Zhang H, et al. Electrochemical synthesis, deposition, and doping of polycyclic aromatic hydrocarbon films. J Am Chem Soc, 2021, 143: 2682-2687

32 Zhang Q, Dong $\mathrm{H}, \mathrm{Hu} \mathrm{W}$. Electrochemical polymerization for twodimensional conjugated polymers. J Mater Chem C, 2018, 6:
10672-10686

33 Gu C, Huang N, Chen Y, et al. $\pi$-Conjugated microporous polymer films: Designed synthesis, conducting properties, and photoenergy conversions. Angew Chem Int Ed, 2015, 54: 13594-13598

$34 \mathrm{Gu} \mathrm{C}, \mathrm{Fei} \mathrm{T}, \mathrm{Lv} \mathrm{Y}$, et al. Color-stable white electroluminescence based on a cross-linked network film prepared by electrochemical copolymerization. Adv Mater, 2010, 22: 2702-2705

35 Chen Q, Luo M, Hammershøj P, et al. Microporous polycarbazole with high specific surface area for gas storage and separation. J Am Chem Soc, 2012, 134: 6084-6087

36 Friebe C, Hager MD, Winter A, et al. Metal-containing polymers via electropolymerization. Adv Mater, 2012, 24: 332-345

37 Zhu Y, Zhang J, Chen Z, et al. Synthesis of nitrocarbazole compounds and their electrocatalytic oxidation of alcohol. Chin J Catal, 2016, 37: 533-538

38 Kortekaas L, Lancia F, Steen JD, et al. Reversible charge trapping in bis-carbazole-diimide redox polymers with complete luminescence quenching enabling nondestructive read-out by resonance Raman spectroscopy. J Phys Chem C, 2017, 121: 14688-14702

39 Qin L, Ma W, Hanif M, et al. Donor-node-acceptor polymer with excellent $\mathrm{n}$-doped state for high-performance ambipolar flexible supercapacitors. Macromolecules, 2017, 50: 3565-3572

40 Song Z, Zhan H, Zhou Y. Polyimides: Promising energy-storage materials. Angew Chem Int Ed, 2010, 49: 8444-8448

41 Li C, Xue J, Huang A, et al. Poly( $N$-vinylcarbazole) as an advanced organic cathode for potassium-ion-based dual-ion battery. Electrochim Acta, 2019, 297: 850-855

42 Zhu L, Ding G, Xie L, et al. Conjugated carbonyl compounds as high-performance cathode materials for rechargeable batteries. Chem Mater, 2019, 31: 8582-8612

43 Jiang Q, Xiong P, Liu J, et al. A redox-active 2D metal-organic framework for efficient lithium storage with extraordinary high capacity. Angew Chem Int Ed, 2020, 59: 5273-5277

44 Xiong $\mathrm{P}$, Yin $\mathrm{H}$, Chen $\mathrm{Z}$, et al. Thiourea-based polyimide/RGO composite cathode: A comprehensive study of storage mechanism with alkali metal ions. Sci China Mater, 2020, 63: 1929-1938

Acknowledgements This work was supported by the National Natural Science Foundation of China (51672188 and 52073211).

Author contributions Wang $\mathrm{W}$ conceived the ideas and performed the experiments and characterizations. Zhao C, Xiong $\mathrm{P}$ and $\mathrm{Su} \mathrm{H}$ provided support for experimental feasibility. Xu Y supervised the project. Wang $\mathrm{W}$ wrote the paper; Yang J and $\mathrm{Xu} \mathrm{Y}$ revised the paper. All authors contributed to the interpretation of the results and approved the final manuscript.

Conflict of interest The authors declare that they have no conflict of interest.

Supplementary information Supporting data are available in the online version of the paper. 


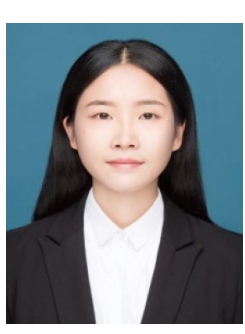

Wei Wang received her BSc degree from the Northeastern University in 2018. She is now a postgraduate under the supervision of Prof. Yunhua Xu at the School of Materials Science and Engineering, Tianjin University. Her current research mainly focuses on the synthesis and characterization of organic cathode materials for electrochemical energy storage devices.

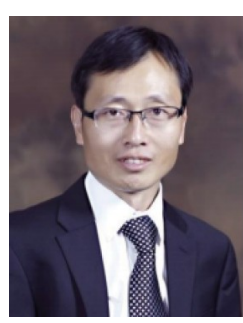

Yunhua Xu is a professor at the School of Materials Science and Engineering, Tianjin University. He received $\mathrm{PhD}$ degree in materials physics and chemistry from the South China University of Technology in 2008. Prior to joining Tianjin University, he worked as a visiting student and postdoc at the University of California, Santa Barbara, Iowa State University and the University of Maryland, College Park, from 2006 to 2015. His research interests focus on electrochemical storage materials and devices.

\section{原位电化学聚合方法制备稳定且高倍率的 锂离子电池双极性有机正极材料}

王微, 赵晨, 杨继兴, 熊佩勋, 苏海, 许运华 ${ }^{*}$

摘要 为了解决有机电极材料的溶解问题并提高电化学性能, 本文 通过原位电化学聚合的方法制备了双极性对氨基苯基咔唑菜二酰 亚胺(APCNDI)有机正极材料. 该正极由能够储存锂离子的 $\mathrm{n}$ 型 1,4,5,8-菜四甲酰基二酰亚胺基团和吸附阴离子的p型的咔唑基团 组成, 其中咔唑基团同时可以充当电化学聚合反应位点. 由原位电 化学聚合方法制备的该正极材料有效解决了有机材料在电解质中 的溶解问题, 表现出双极性特征的氧化还原反应, 并具有出色的倍 率性能和稳定的循环性能. 该有机正极材料循环 1000 次后的容量 仍能保持为 $92 \mathrm{~m} \mathrm{~h} \mathrm{~h}^{-1}$, 在 $10 \mathrm{~A} \mathrm{~g}^{-1}$ 的大电流密度下比容量剩余 $72 \mathrm{~mA} \mathrm{~h} \mathrm{~g}^{-1}$. 同时, 通过多种表征手段对APCNDI正极材料的双极 性特征和反应机理进行了系统的研究和验证. 该发现为发展高性 能的有机电极材料提供了一种新颖的设计思路和制备方法. 